\title{
Effect of Naturally Occurring E2F-4 Alterations on Transcriptional Activation and Proliferation in Transfected Cells
}

\author{
Hirotoshi Takashima, Yusuke Matsumoto, Nagahide Matsubara, \\ Yasuhiro Shirakawa, Ryuichi Kawashima, Motohiko Tanino, Sachio Ito, \\ Hiroshi Isozaki, Mamoru Ouchida, Stephen J. Meltzer, Kenji Shimizu, and \\ Noriaki Tanaka
}

Department of Gastroenterological Surgery and Surgical Oncology (HT, YM, NM, YS, RK, HI, NT), and Department of Molecular Genetics (NM, MT, SI, MO, KS), Okayama University Graduate School of Medicine, Okayama, Japan; Department of Medicine (GI Division) and Greenebaum Cancer Center (SJM), University of Maryland School of Medicine and Baltimore Veterans Administration Hospital, Baltimore, Maryland

SUMMARY: E2F is a family of transcription factors implicated in the regulation of gene expression required for progression through the $\mathrm{G}_{1}-\mathrm{S}$ transition. We have previously detected tumor-specific mutations at a trinucleotide repeat coding sequence of E2F-4 gene in a subset of human sporadic colorectal cancers. The purpose of this study was to investigate the potential functional consequences of these E2F-4 mutations. We transfected NIH3T3 fibroblasts with expression constructs containing wild-type as well as mutant $E 2 F-4 \mathrm{cDNA}$, and the effect of the $E 2 F-4$ mutations on proliferation was examined. Alteration in transactivation of the E2F consensus promoter sequence was also examined by transient cotransfection of a E2F-4 with a $D P-2$ construct into cultured human cells. Transfected cell clones overexpressing mutant E2F-4 grew more rapidly and showed higher proliferative activity by increased immunohistochemical staining for proliferating cell nuclear antigen (PCNA). All three mutant forms of E2F-4 showed elevated transactivation of the E2F consensus promoter sequence. Thus, expression of mutant E2F-4s confers a growth advantage in vivo, and this effect may be related to the acquisition of a neoplastic phenotype. (Lab Invest 2001, $81: 1565-1573)$.

$T$

he E2F family of transcription factors (E2F-1, -2, $-3,-4,-5$, and -6 ) has been implicated in the regulation of cell cycle, particularly at the level of $G_{1}-S$ progression, as well as in the regulation of apoptotic cell death (Adams and Kaelin, 1996; Cartwright et al, 1998; Krek et al, 1995; La Thangue, 1996; Shan et al, 1996; Weinberg, 1996). E2F-4, one of six members of this gene family, is involved in the regulation of early $\mathrm{G}_{1}$ events, including the $\mathrm{G}_{0} / \mathrm{G}_{1}$ transition, and it facilitates transactivation of genes essential for cellular proliferation (Beijersbergen et al, 1994). In cooperation with DP-2, E2F-4 activates transcription from a promoter that harbors a consensus E2F-binding site. This site is present in the promoters of many growthresponsive and growth-promoting genes, including

Received August 15, 2001.

This work was supported by a Grant-in Aid from the Japanese Ministry of Education, Science, Sports and Culture of Japan (11671237, 11671240, and 10470040), and National Institutes of Health Grants CA85069, DK47717, CA77057, CA78843, and the Medical Research Service, Department of Veterans Affairs.

Address reprint requests to: Dr. Nagahide Matsubara, Department of Gastroenterological Surgery and Surgical Oncology, Okayama University Graduate School of Medicine, 2-5-1 Shikata-cho, Okayama 700-8558, Japan.E-mail:nagamb@cc.okayama-u.ac.jp c-myc, DNA polymerase- $\alpha$, cyclin-dependent kinases, and cyclin D1 (Herber et al, 1994; Pearson et al, 1991; Sardet et al, 1995; Vairo et al, 1995). E2F-4 regulates these genes, in part, through their interaction with members of the pocket proteins (pRb, p130, and p107) (Li et al, 1997; Liu et al, 1998; Sardet et al, 1995; Wells et al, 1997). An important role for E2Fs in the development of cancer is suggested by the finding that, in most human neoplasia, genetic or epigenetic alterations occur that ultimately result in the deregulation of E2F-dependent transcription. Therefore, it is likely that the dysfunction of E2Fs, in cooperation with dysfunction of other proteins, contributes to human malignancy.

We recently identified colorectal tumor-specific mutations in the trinucleotide (AGC) repeat sequence within open reading frame of $E 2 F-4$, which encodes 13 consecutive serine residues (Ikeda et al, 1998; Yoshitaka et al, 1996). Our group also discovered identical E2F-4 mutations in gastric cancers and ulcerative colitis-related neoplasms (Souza et al, 1997). Because E2F-4 is known to be important in cell-cycle control, we reasoned that mutations in E2F-4 conferred a growth advantage relative to wild-type E2F-4. Accordingly, we stably transfected NIH3T3 fibroblasts with expression constructs containing wild-type as well as 
mutant alleles of E2F-4 under transcriptional control of the human $\mathrm{SR} \alpha$ promoter. We have also examined the transactivation of an E2F consensus promoter sequence by mutant forms of E2F-4.

\section{Results}

\section{Establishment of E2F-4 Expressing Cell Lines}

To directly assess the effects of mutant E2F-4 gene expression on cell growth, we constructed expression plasmids by placing the entire coding region of human wild-type and mutant E2F-4 cDNAs under the transcriptional control of $\mathrm{SR} \alpha$ promoter. We stably transfected these constructs into NIH3T3 cells. DNA was extracted from each antibiotic selected clone, and the correct insertion of each plasmid was confirmed by
PCR and sequencing with the primer set we previously described (Yoshitaka et al, 1996) (Fig. 1A). Northern blot analysis of wild-type and mutant E2F-4 mRNA in transfected NIH3T3 cells was performed in three separate experiments, revealing that all the transfected cell lines overexpressed human E2F-4 mRNA (Fig. 1B). Virtually no expression was detected in cells transfected with control empty vector pCDL81. As shown in Fig. 1C, high level expression of E2F-4 proteins was detected by Western blotting in all mutant cells compared with wild-type cells.

The influence of E2F-4 mutations on cell morphology was monitored by phase-contrast light microscopy. These cell lines exhibited increasing saturation density and lack of contact inhibition compared with the cell lines expressing wild-type E2F-4 or empty

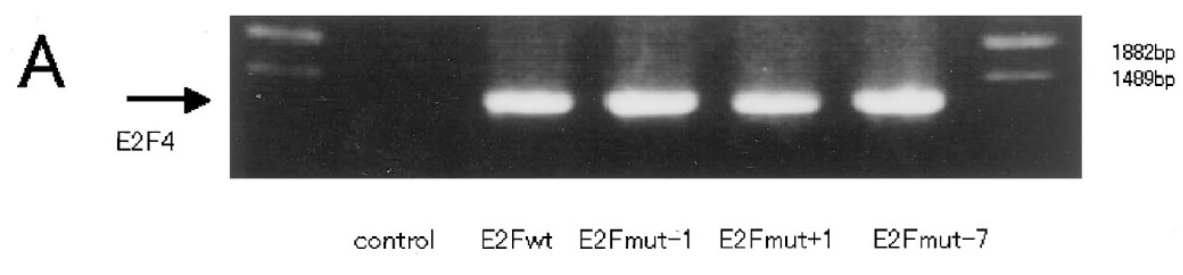

B

E2F4

GAPDH
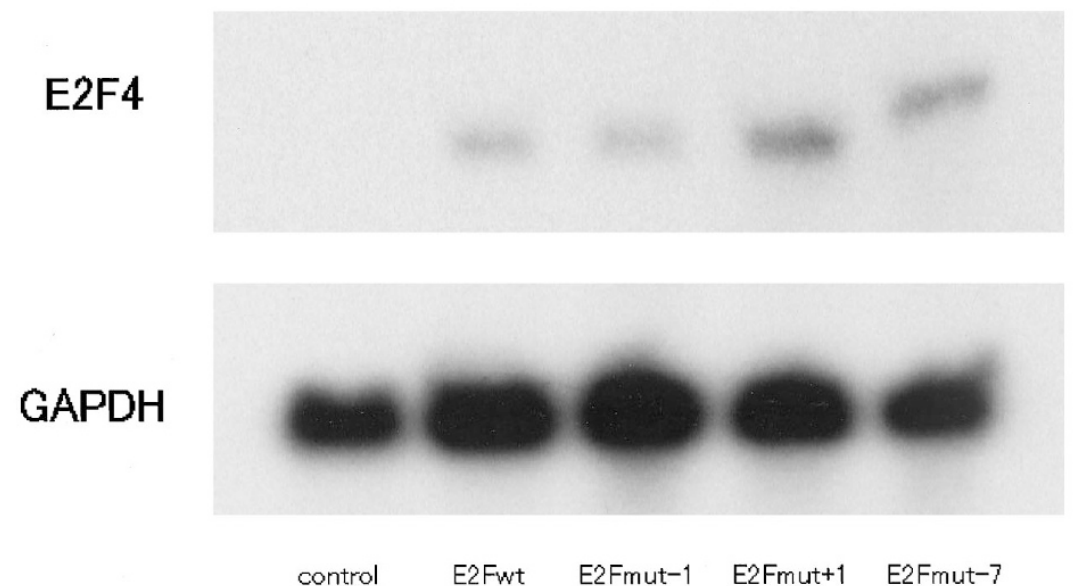

C

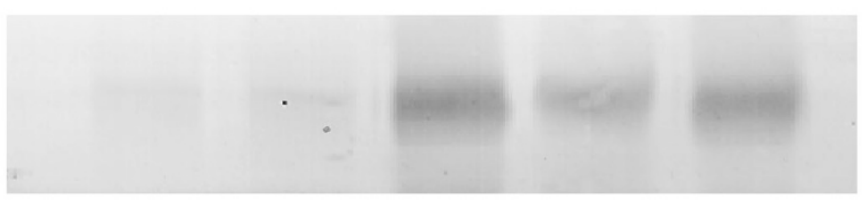

control

E2Fwt

E2Fmut-1 E2Fmut+1

E2Fmut-7

Figure 1.

A, PCR using the specific primer set for inserted human E2F-4s was performed to confirm the correct insertion of each vector into the transfected cell lines. B, Northern blot analysis of E2F-4 mRNA with or without mutations in transfected NIH3T3 cells. Total RNA from each cell was isolated, fractionated by electrophoresis in a denaturing agarose gel, transferred to a nylon membrane, and hybridized to ${ }^{32}$ P-radiolabeled human E2F-4 cDNA. The filter was stripped and reprobed with glyceraldehyde-3-phosphate dehydrogenase (GAPDH) cDNA. All the transfected cells overexpressed human E2F-4 mRNAs. C, Western blot analysis of NIH3T3 cells transfected with wild-type or mutant E2F-4s. The mutant E2F-4 cells (E2Fmut ${ }^{-1}$, E2Fmut ${ }^{+1}$, and E2Fmut ${ }^{-7}$ ) showed overexpression of E2F-4 protein, whereas the wild-type E2F-4 (E2Fwt) cells showed a low level of E2F-4 expression. 
vector. However, none of the cell lines expressing mutant E2F-4 acquired the ability to grow in soft agar (data not shown). We also tested the effect of E2F-4 mutations on tumorigenicity by subcutaneous injection of these transfected cell lines $\left(5 \times 10^{6}\right.$ each in 0.2 $\mathrm{ml}$ ) into the lower flank of 2-month-old female nude mice. No tumor was developed in nude mice within 8 weeks of the inoculation of these cell lines (data not shown).

\section{Growth Properties of Clones Expressing Mutant E2F-4s}

The cell lines that expressed wild-type or mutant $E 2 F-4 s$ were studied for several aspects of growth regulation. All three mutant E2F-4 genes manifested significant changes in growth properties compared with cells expressing wild-type E2F-4. Cells transfected with mutant E2F-4s proliferated more rapidly than those transfected with wild-type E2F-4 or control vector (Fig. 2). Notably, the mutant E2F-4 containing a deletion of one unit AGC (E2Fmut ${ }^{-1}$ ), which is the most prevalent mutation in human cancer, exhibited the most profound effect on cell proliferation. Proliferating cell nuclear antigen (PCNA) immunohistochemical staining also confirmed high proliferative activity of NIH3T3 cells transfected with mutant E2F-4, including E2Fmut $^{-1}$, E2Fmut ${ }^{+1}$, and E2Fmut ${ }^{-7}$ (Fig. 3, B and C).

\section{Immunohistochemical Staining of E2F-4}

The difference in expression of the E2F-4 protein was examined on NIH3T3 cells stably transfected with

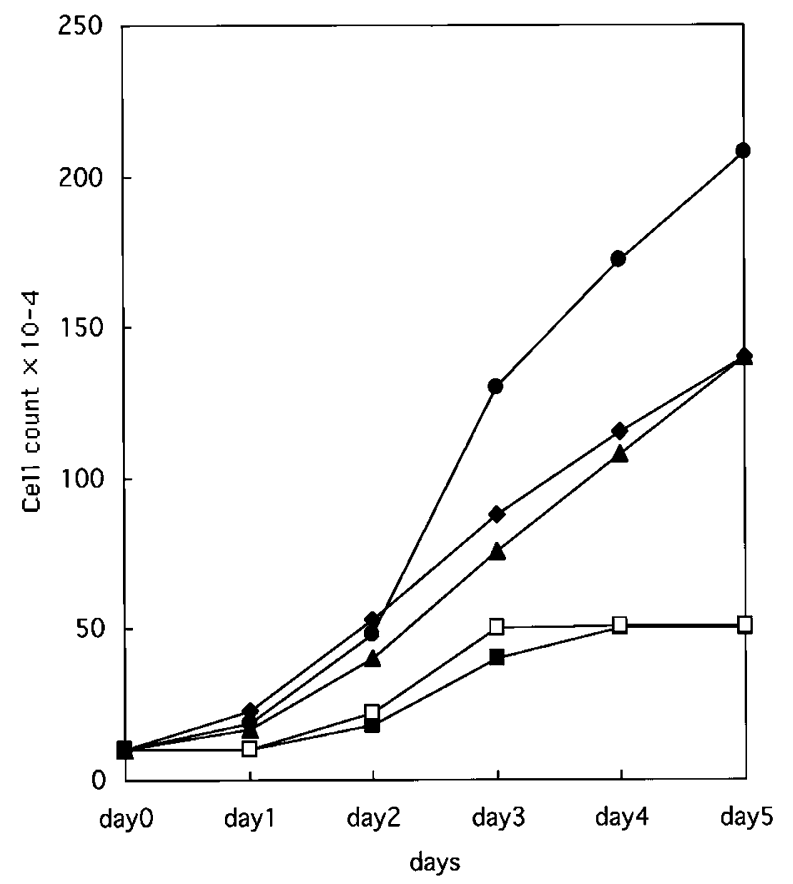

Figure 2.

Growth of the NIH3T3 cells transfected with wild-type and mutant types of E2F-4 cDNA in media with a high serum concentration. $\square$, NIH3T3 cells transfected with control vector; $\bullet$, E2Fmut ${ }^{-1} ; \boldsymbol{\Delta}$, E2Fmut $^{+1} ; \diamond$, E2Fmut $^{-7}$; $\square$, E2Fwt. Cells were counted at the indicated times with a Coulter cell counter. All measurements were made in triplicate, and the standard deviation of each point was within the symbol. wild-type as well as mutant E2F-4 cDNA-expressing vector. Especially intracellular localization of E2F-4 protein was evaluated (Fig. 3A). In mock transfected cells and in wild-type E2F-4 transfected cells, E2F-4 protein were predominantly stained in cytosol. On the other hand, predominant nuclear localization of E2F-4 protein was observed in mutant cells of E2Fmut ${ }^{-1}$, $\mathrm{E}_{2} \mathrm{Fmut}^{+1}$, and E2Fmut ${ }^{-7}$. The percentage of the transfected cells revealing nuclear localization of E2F-4 protein among all cells was determined. The nuclear localization of mutant E2Fmut ${ }^{-1}$, E2Fmut ${ }^{+1}$, and $\mathrm{E} 2 \mathrm{Fmut}{ }^{-7}$ cells showed $65 \%, 63 \%$, and $47 \%$, respectively, whereas wild-type E2Fwt showed only $32 \%$. Localization of pocket proteins in wild-type as well as mutant cells was also assessed by immunohistochemical staining (Fig. 4, A and C). Increased nuclear staining of p107-positive cells was observed in the most prevalent forms of the mutant cells, including E2Fmut ${ }^{-1}$ and E2Fmut ${ }^{+1}$, but not in E2Fmut $^{-7}$ cells (Fig. 4B). On the other hand, nuclear staining of p130 was not elevated in mutant or wildtype cells (Fig. 4D).

\section{Flow Cytometry Analysis}

Flow cytometry analysis was performed on NIH3T3 cells transfected with wild-type, as well as mutant E2F-4, including E2Fwt, E2Fmut ${ }^{-1}, \mathrm{E}_{2} \mathrm{Fmut}^{+1}$, and $\mathrm{E}_{2} \mathrm{Fmut}^{-7}$. The cells were synchronized by serum starvation and were released by refeeding with FCS (Fig. 5A). After 16 hours post-synchronization, three of the mutant cells entered S-phase, but wild-type cells had not entered S-phase, suggesting a shorter cellcycle interval of the mutant cells compared with the wild-type E2F-4 expressing cells (Fig. 5B).

\section{Transcription Activity of Wild-Type and Mutant E2F-4}

One of functions ascribed to E2F is to bind to E2F consensus sites and mediate transactivation of the adenovirus E2 promoter in an E1A-dependent manner. Therefore, we tested the ability of mutant E2F-4s to transactivate the E2 promotor. SAOS.2 cells were co-transfected with an E2F-4 expression vector, pCDL81/DP-2, and a reporter plasmid containing E2F DNA-binding sites. Co-transfection of E2F-4 with DP-2 was performed because E2F-4 may preferentially associate with DP-2 rather than DP-1 (Magae et al, 1996). Surprisingly, all mutant cells expressed 6- to 8-fold increase in the level of luciferase expression arising from the E2 reporter construct (Fig. 6A). These results suggest that mutations in the $A G C$ repeat sequence increased the transactivation ability of the gene product. We confirmed the results with the repeated experiments. We also performed an identical experiment on HEK-293 cells. Again, only cells introduced with mutant E2F-4 constructs showed a 2-fold increase in the level of luciferase expression (Fig. 6B).

\section{Discussion}

Although the tumor-specific alterations of E2F-4 in trinucleotide $(A G C)$ repeat sequence within the open 
A
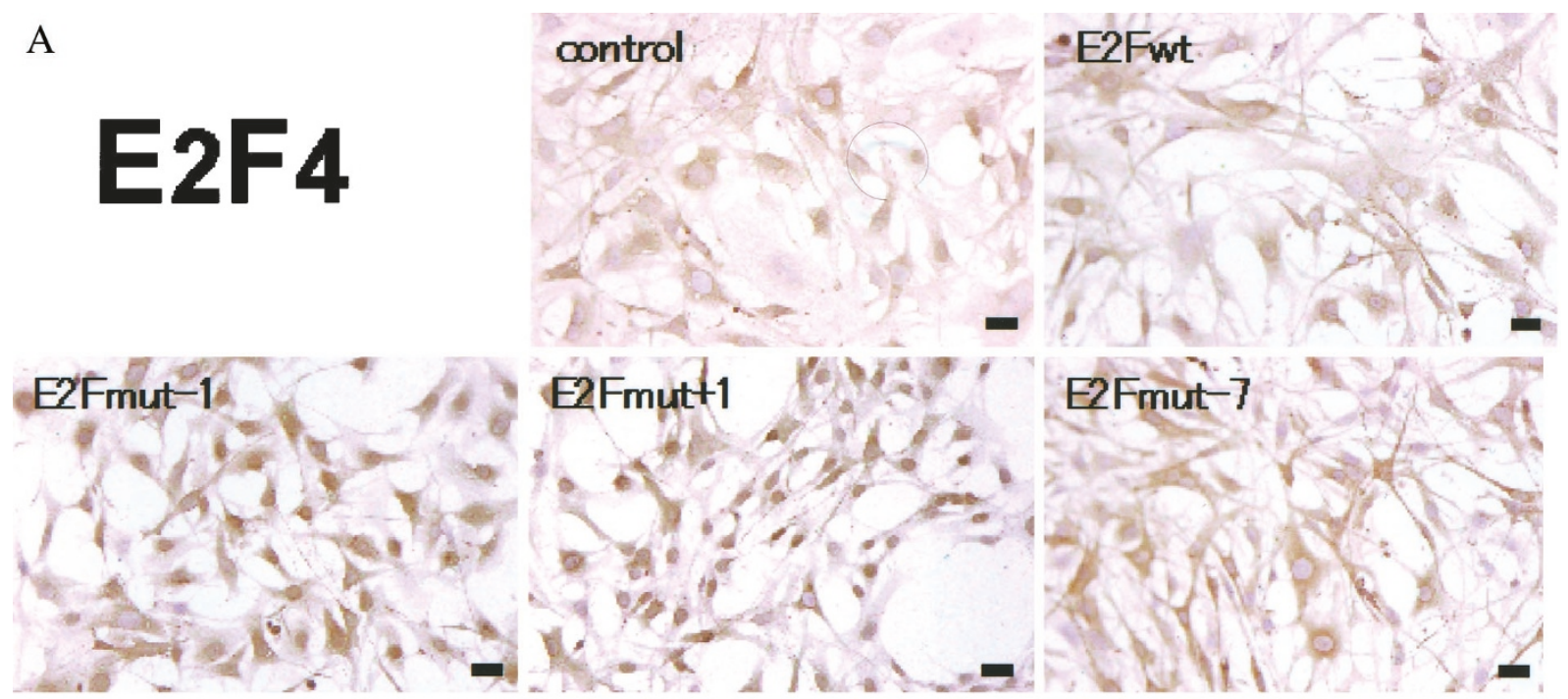

\section{E2Fmutt1}

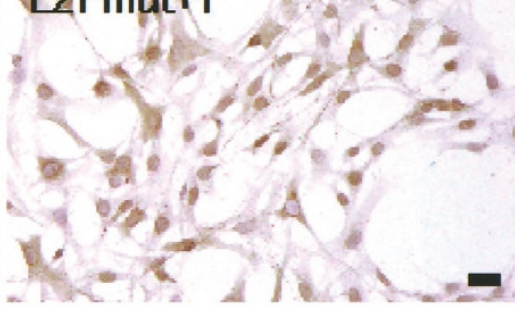

B
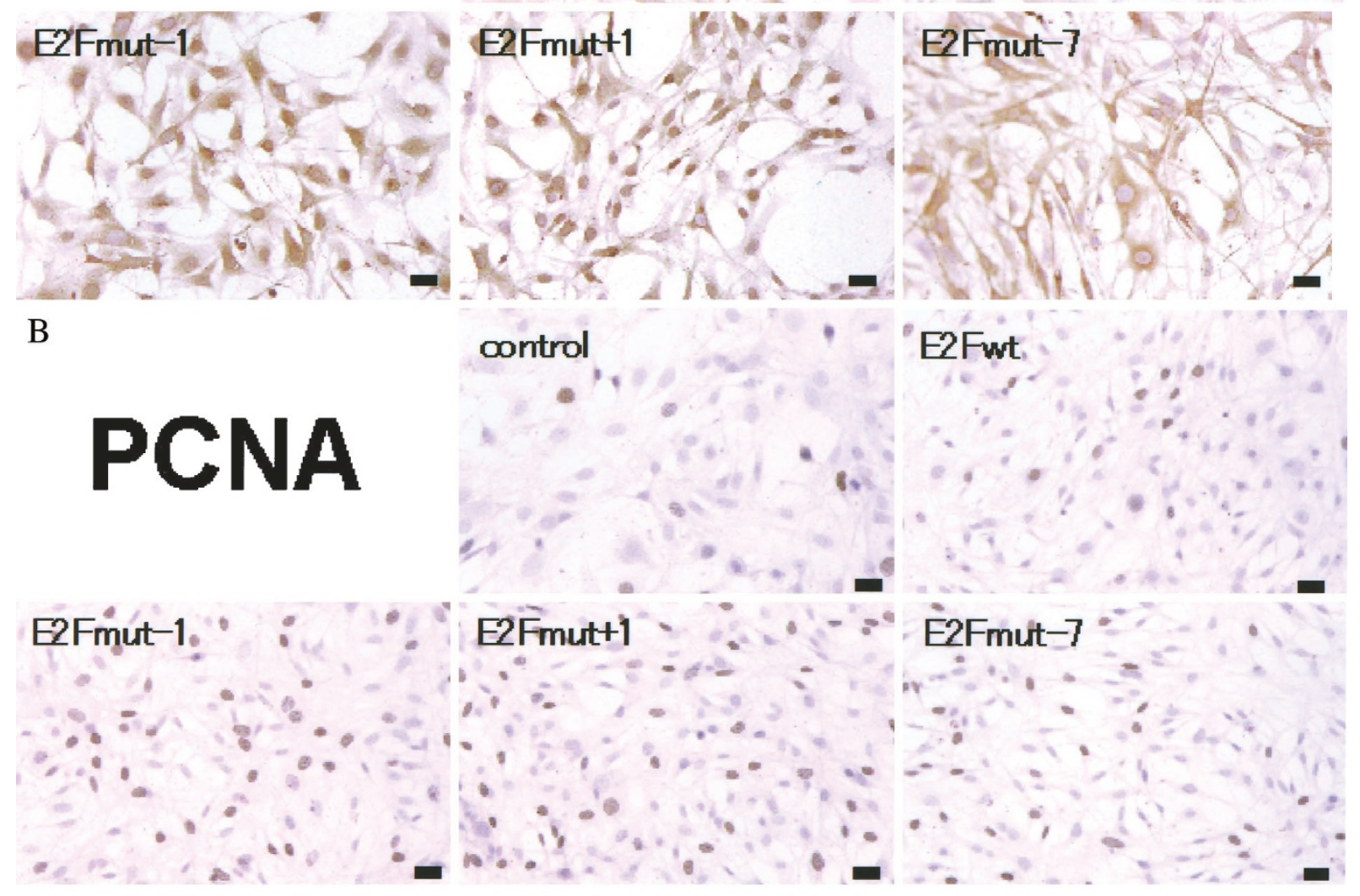

C

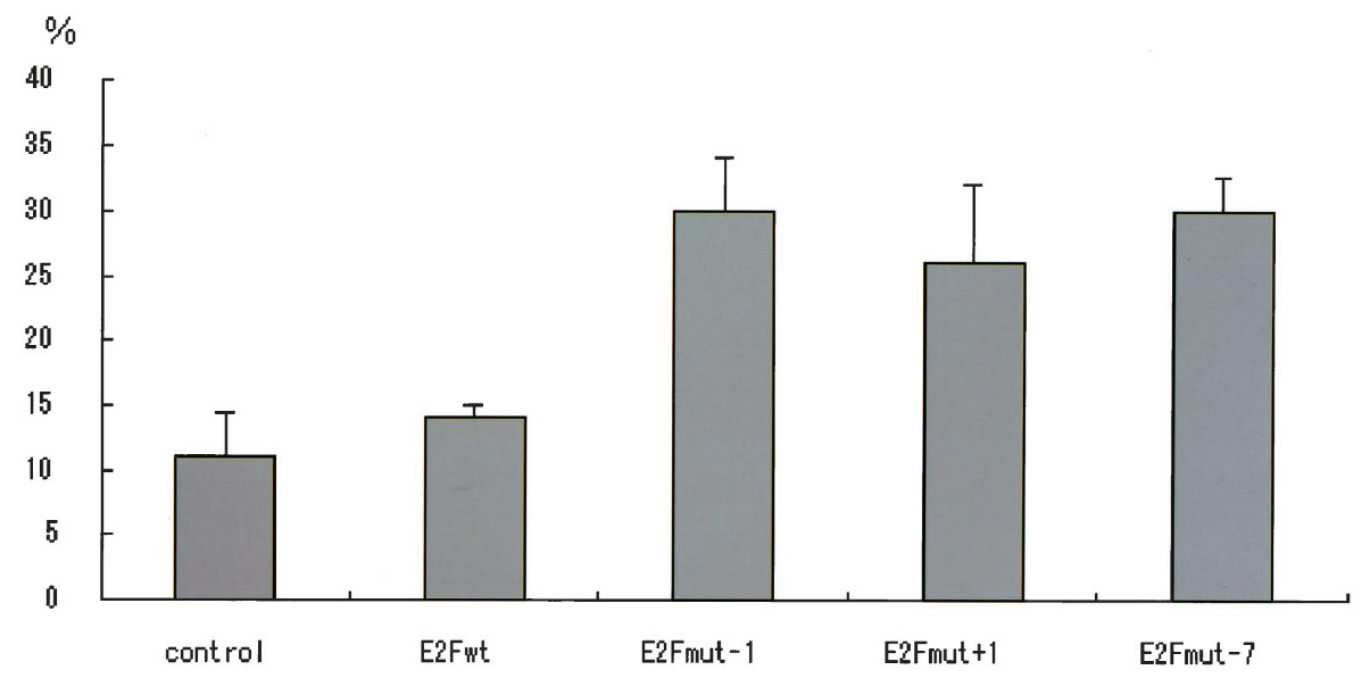

Figure 3.

A, Immunohistochemical staining for E2F-4 on mutant and wild-type NIH3T3 cells. B. Immunohistochemical staining for proliferating cell nuclear antigen (PCNA) on the same NIH3T3 cell lines. C, PCNA staining was quantitated by counting the number of PCNA-positive cells per 100 cells in control or E2F-4 transfected cells. 
A

A

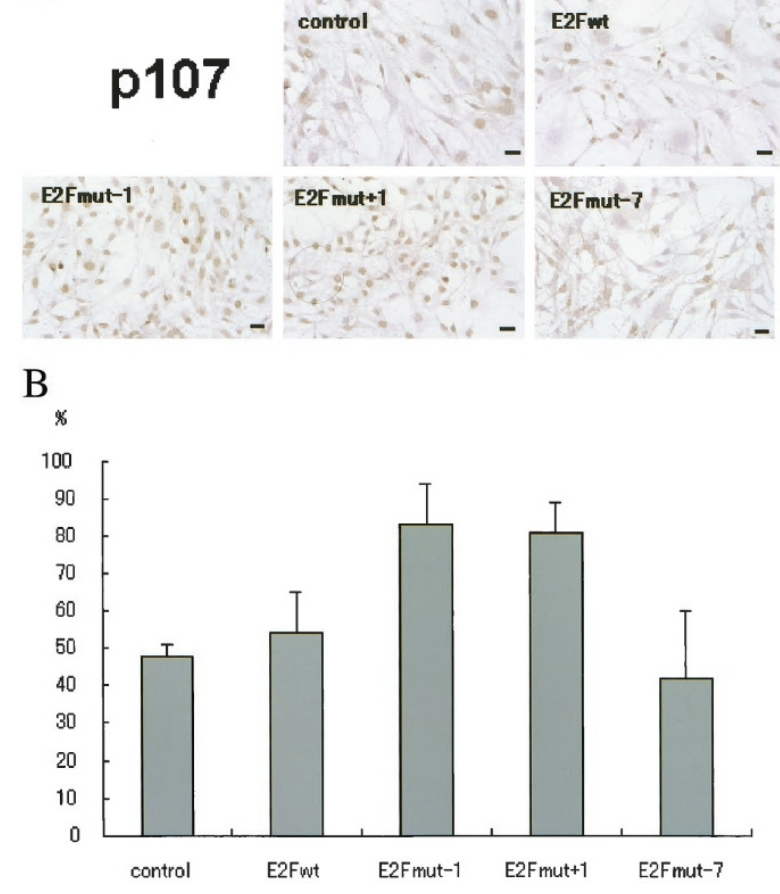

$\mathrm{C}$

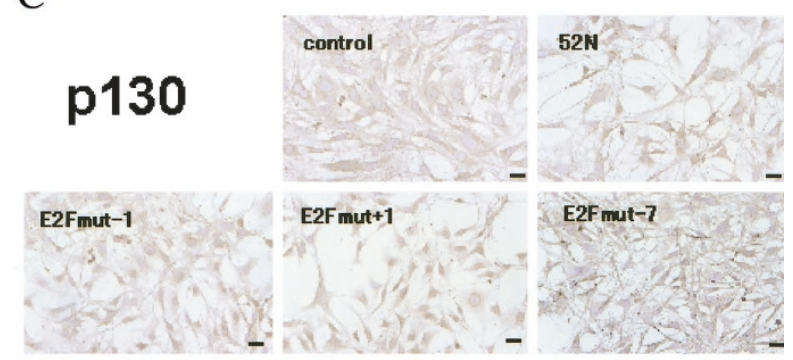

$\mathrm{D}$

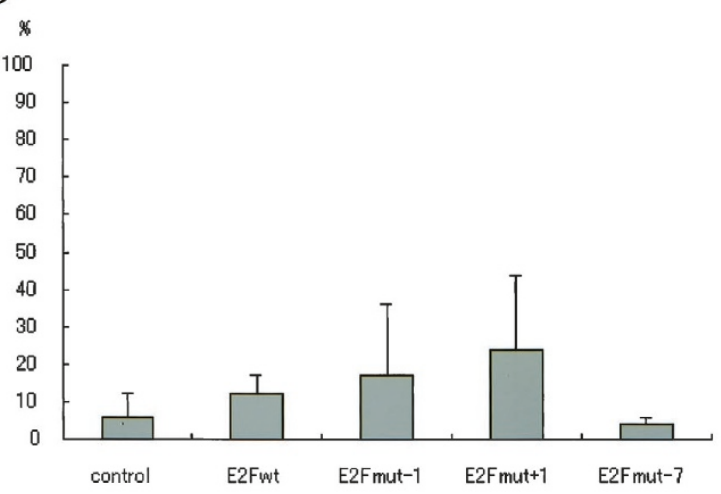

Figure 4.

A, Immunohistochemical staining for p107 on mutant and wild-type NIH3T3 cells. B, Nuclear localization of p107 was quantitated by counting the number of nuclear-stained, p107-positive cells per 100 cells in control or E2F-4 transfected cells. C, Immunohistochemical staining for p130 on mutant and wild-type NIH3T3 cells. D, Nuclear localization of p130 was quantitated by counting the number of nuclear-stained, p130-positive cells per 100 cells in control or E2F-4 transfected cells.

reading frame was identified in gastrointestinal cancers, the consequence of E2F-4 alterations was not known. In the present study, the properties of the E2F-4 alterations detected in such cancers were investigated in transfected cells.

Our results showed that the exogenous mutant E2F-4 species expressed in transfected NIH3T3 cells produced significant changes in growth properties. Increased PCNA-positive staining and rapid entry into S-phase by flow cytometry analysis also demonstrated the increased proliferation in E2F-4 mutant cells. These results support the hypothesis that E2F-4 mutation leads to increased cell proliferation. To avoid the considerable possible clonal variations, we picked 5 to 10 clones per construct and confirmed that all cells having the same construct showed the same properties. We confirmed that these same properties were also observed in other clones possessing identical mutations, which were established in the same transfection/selection process.

At a mechanistic level, the functional significance of E2F-4 mutation was shown by transient cotransfection of mutant E2F-4s and DP-2 into human SAOS. 2 or HEK-293 cells. Specifically, mutations in the AGC repeat sequence lead to up-regulation of the reporter construct containing consensus E2F binding sites in these cells. Although the transfection of mutant E2F-4 without DP-2 resulted in elevated transactivation of the reporter gene, the variable of each experiment was inconsistent for unknown reasons. Thus, co-transfection of E2F-4s and DP-2 was shown. The difference in E2F-4 protein localization within the transfected cells affected by mutations of E2F-4 was also observed. It is known that E2F-1 to 3 localize in the nucleus and preferentially bind to $\mathrm{pRb}$, whereas E2F-4 and 5 have no nuclear localization signal (NLS) and preferentially bind to p107/p130. Transcriptional activation required translocation of the E2F-4 protein into the nucleus, perhaps by DP-2, a heterodimeric partner that has a NLS (de la Luna et al, 1996; Lindeman et al, 1997; Muller et al, 1997; Verona et al, 1997). Our experiments showed that more nuclei localization of E2F-4 protein was observed in E2F-4 mutant cells than in the wild-type cells. This may also explain the elevated transactivation ability induced by mutant E2F-4 genes. As shown in the immunohistochemical study of p107 and p130, more nuclear localization of $\mathrm{p} 107$ was observed in the most prevalent mutant cells, including E2Fmut ${ }^{-1}$ and E2Fmut ${ }^{+1}$, than in wild-type cells, suggesting that p107 may contribute to the nuclear translocation of mutant E2F-4 into the nucleus in cooperation with DP-2.

E2Fs regulate the expression of a number of genes important to cell-cycle progression, particularly those involved in progression through $\mathrm{G}_{1}$ into the $\mathrm{S}$-phase of the cell cycle. The activity of E2F factors is regulated through association with the pocket family proteins. The interplay among $\mathrm{G}_{1}$ cyclins (D-type cyclins and cyclin E), cyclin-dependent kinases (cdk4, 6, and 2), cdk inhibitors, and protein phosphatases determines the phosphorylation status of the pocket proteins. This phosphorylation status, in turn, regulates the ability of the pocket proteins to complex with E2F's. The binding of pocket proteins to E2F converts E2F factors 

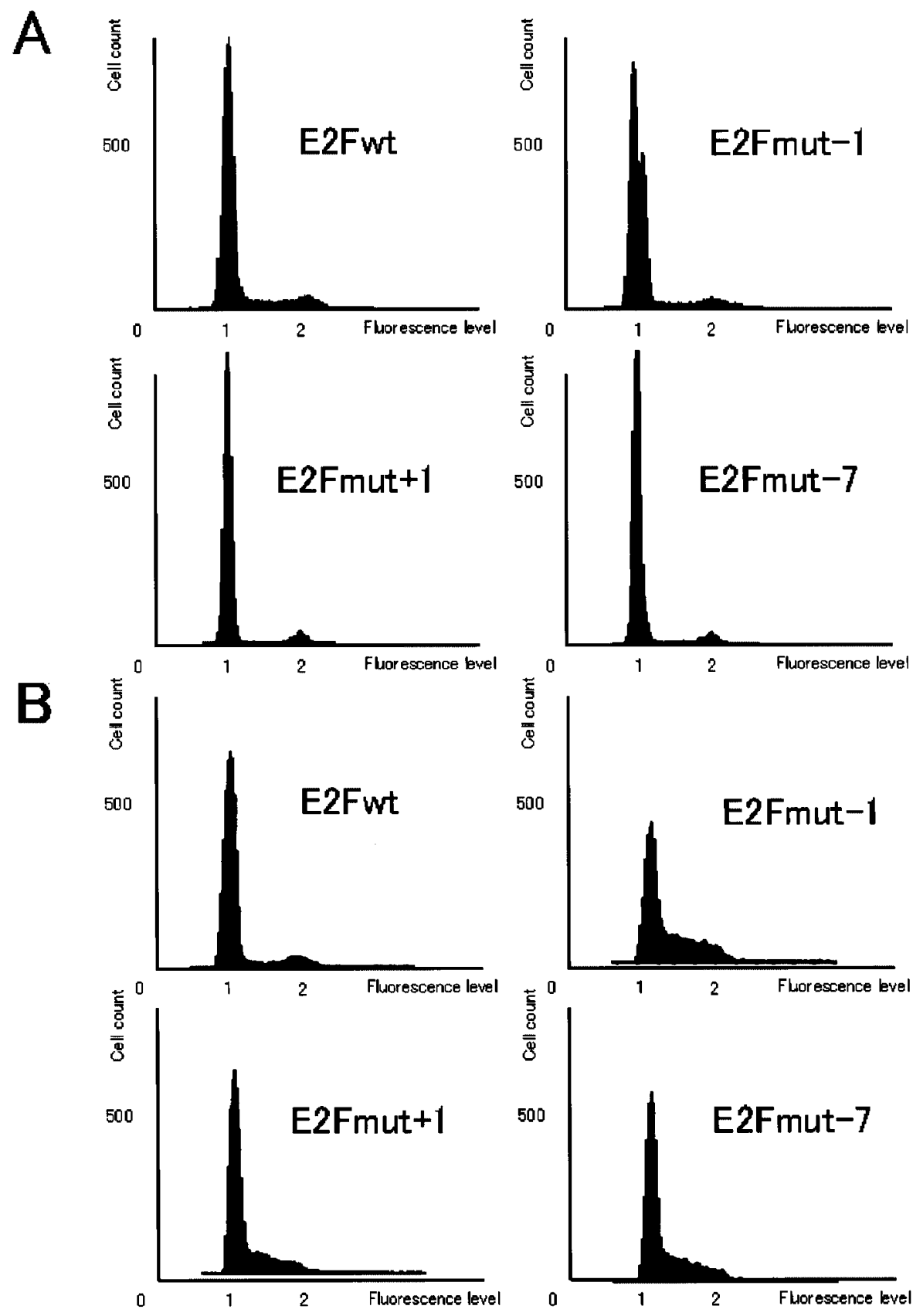

Figure 5.

The effect of ectopic expression of mutant and wild-type E2F-4 on cell-cycle progression. A, Cells were arrested in $G_{0} / G_{1}$ by serum starvation followed by release with fresh medium containing $5 \%$ FCS. B, Sixteen hours post-synchronization, three of the mutant cells entered S-phase, but wild-type cells had not entered S-phase.

from transcriptional activators into transcriptional repressors (Weintraub et al, 1992). The fact that E2F-4 represses transcription when bound to pocket proteins may suggest that its main physiologic role is to inhibit growth-promoting genes such as the tumor suppressor genes.

The E2F-4 serine repeat domain, which may function in transactivation of other growth-stimulatory genes (Sardet et al, 1995), lies between the "marked box" region and the pocket protein binding region. It is possible that mutations in the serine repeat domain contribute to tumorigenesis by inducing a conforma- tional change in the protein that interferes with binding of pocket proteins. This theory would suggest that increased levels of free E2F-4 occur in transformed cells as a result of their dissociation from pocket proteins.

In recent years, however, it has become apparent that the role of E2F is not exclusively in activation. Several genes expressed in late $\mathrm{G}_{1}$ /early $\mathrm{S}$, including $B-m y b, D H F R$, and E2F-1, have been shown to be repressed through an E2F-mediated mechanism in $\mathrm{G}_{0} / \mathrm{G}_{1}$ and to be derepressed in late $\mathrm{G}_{1}$. Therefore, it may also be possible that E2F-4 mutation in the serine 

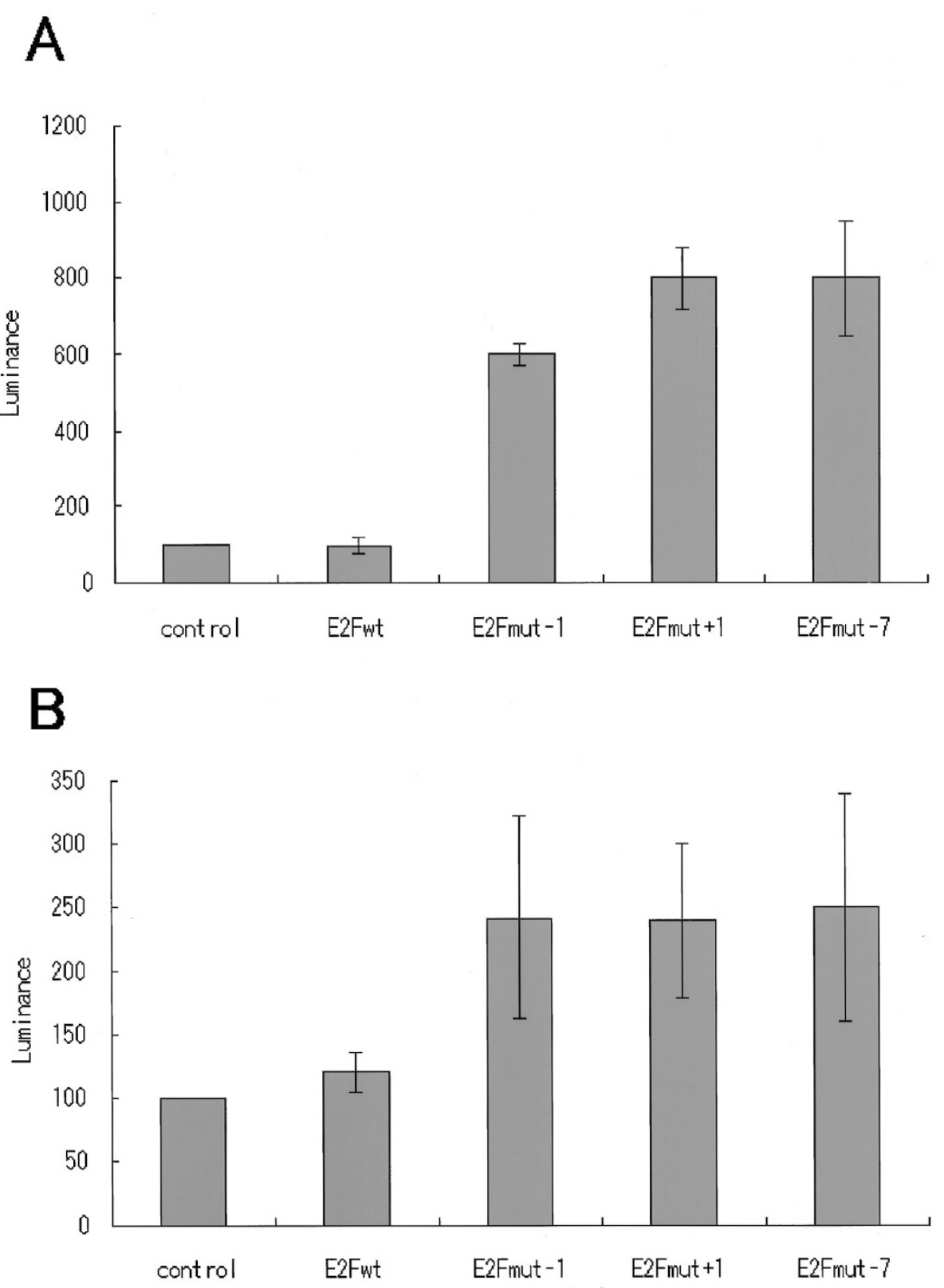

Figure 6.

Luciferase reporter assay. A, Coexpression of mutant E2F-4s and DP-2 in SAOS.2 cells results in increased transactivation of a luciferase reporter gene compared with the control or wild-type E2F-4 expression vector cotransfected with DP-2 expression vector. B, Coexpression of mutant E2F-4s and DP-2 in HEK-293 cells. Increase transactivation of luciferase activity was also observed in mutant cells.

repeat domain disturbs this repression function, resulting in dysregulated gene transactivation.

In summary, we report that mutant E2F-4 detected in primary gastrointestinal cancers cause elevated transactivation of E2F consensus promoter sites and alter the growth properties of cells. It may be reasonable to expect that the mutation of E2F-4 contributes to neoplastic progression.

\section{Materials and Methods}

\section{Cell Lines and Culture Conditions}

The NIH3T3 fibroblast cell line was obtained from the American Type Culture Collection (Manassas, Virginia) and grown in DMEM supplemented with $2 \mathrm{~mm}$ L-glutamine and 10\% FCS. SAOS.2, a human osteosarcoma cell line, and HEK-293, a human embryonal kidney cell line, were grown in DMEM supplemented with $10 \%$ FCS.

\section{Cloning of E2F-4 in a Mammalian Expression Vector and Transfection of NIH3T3 Cells}

The human E2F-4 cDNA from the total RNA of the colorectal cancer and normal mucosa from the patients in our previous report (Yoshitaka et al, 1996) was constructed by reverse transcription-polymerase chain reaction (RT-PCR) and cloned into the pBlue- 
script KS(-) (Stratagene, La Jolla, California). The resultant vectors were different in their (AGC) repeat number, consisting of wild-type E2F-4 with (AGC) $)_{13}$, mutant $E 2 F-4$ with $(A G C)_{12}$, and mutant E2F-4 with (AGC) ${ }_{14}$. We also constructed an E2F-4 mutant having 21 bp deletion, $(A G C)_{6}$, by PCR with specific primers and ligation. Then the resultant E2F-4 cDNAs were excised from the vector with $\mathrm{Xhol}$ and $\mathrm{BamHI}$ and cloned into the Xhol/BamHI site of the pCDL81 expression vector downstream of the human $\mathrm{SR} \alpha$ promoter. The transfection of these expression vectors into NIH3T3 cells was carried out using a calcium phosphate coprecipitation protocol. The cells were grown under selection with hygromycin, and resistant colonies were cloned. All the selected cells were maintained in medium containing $200 \mu \mathrm{g} / \mathrm{ml}$ hygromycin. The resultant cell lines transfected with pCDL81/ wild(AGC) $)_{13}, \quad$ pCDL81/mut(AGC) ${ }_{12}, \quad$ pCDL81/mu-

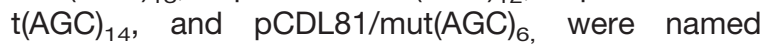
E2Fwt, E2Fmut ${ }^{-1}$, E2Fmut ${ }^{+1}$, and E2Fmut ${ }^{-7}$, respectively. pCDL81/DP2 was also constructed in an analogous manner after the full-length DP-2 cDNA was generated by RT-PCR with specific primers. The integrity of each cDNA was confirmed by sequencing over the entire coding region.

\section{Northern Blot Analysis}

An E2F-4 probe was prepared by restriction endonuclease-digestion of the PCDL81/E2F-4 vector and ${ }^{32} \mathrm{P}$-labeled by random priming. Hybridizations were performed for 16 hours at $42^{\circ} \mathrm{C}$ in a standard method. The final washing of the membranes was performed at $55^{\circ} \mathrm{C}$. Membranes were exposed to X-Omat film at $-80^{\circ} \mathrm{C}$ with Cronex intensifying screens (Dupont, Wilmington, Delaware).

\section{Determination of Growth in Culture}

Approximately $3 \times 10^{6}$ cells of each transfected line were plated on 50-mm dishes in DMEM supplemented with $10 \%$ FCS and $200 \mu \mathrm{g} / \mathrm{ml}$ hygromycin. Cells were counted at the indicated times with a Coulter cell counter (Coulter Electronics, Miami Lake, Florida). All measurements were made in triplicate.

\section{Immunohistochemical Staining Study}

Cells were cultured on tissue culture chamber slides for 48 hours. The slides were fixed in cold $20 \%$ formaldehyde for 10 minutes. Endogenous peroxidase was blocked by incubating the sections in $3 \%$ hydrogen peroxidase in methanol for 10 minutes. After the blocking of nonspecific reactivity for 10 minutes at room temperature with goat serum, the sections were incubated overnight at $4^{\circ} \mathrm{C}$ with mouse monoclonal anti-PCNA antibody (PC-10; Santa Cruz Biotechnology, Santa Cruz, California), rabbit polyclonal antiE2F-4 antibody (C-20, Santa Cruz Biotechnology), rabbit polyclonal anti-p107 (C-18, Santa Cruz Biotechnology), or rabbit polyclonal anti-p130 (C-20, Santa Cruz Biotechnology). The identification of the distribution of primary antibodies was achieved by the subsequent application of a biotinylated secondary antibody and streptavidin-peroxidase. Immunostaining was developed using $\mathrm{DBA} / \mathrm{H}_{2} \mathrm{O}_{2}$ solution, and the sections were counter-stained with Mayer's hematoxylin.

\section{Transient Transfection and Transactivation Assays}

A plasmid having an E2F-consensus promoter, pE2CATwt, was a gift from Joseph R. Nevins, a Howard Hughes Medical Institute investigator at Duke University. A reporter plasmid containing the luciferase gene under control of the E2F-dependent promoter, $p E 2 / L u c i f e r a s e$, was constructed by recombining $\mathrm{pSR} \alpha /$ Luciferase and $\mathrm{pE2CATwt.} \mathrm{For} \mathrm{luciferase}$ reporter assay, cotransfection experiments were done with the variety of $\mathrm{pCDL81/E2F-4} \mathrm{expression} \mathrm{vectors,}$ together with $\mathrm{pCDL81/DP2}$ and reporter pSR $\alpha /$ Luciferase plasmid. Cotransfection of vectors into SAOS.2 cells, as well as HEK-293 cells with these plasmids, was carried out by the methods of Chen and Okayama (1987). Cells were harvested 30 hours after transfection. Cell lysis and luciferase assays were carried out as described elsewhere (Ausubel et al, 1992).

\section{Nude Mouse Assays}

Athymic nude mice were given subcutaneous injections of wild-type and mutant E2F-4 transfected cells (E2Fwt, E2Fmut ${ }^{-1}$, E2Fmut ${ }^{+1}$, and E2Fmut ${ }^{-7}$ ). Each cell was trypsinized and suspended in PBS for injection. Cell suspensions $\left(5 \times 10^{6}\right.$ cells in $\left.0.2 \mathrm{ml}\right)$ were injected subcutaneously into lower flank of 2-monthold female nude mice. Mice were killed 8 weeks later.

\section{Western Blot Analysis}

Whole cell lysate from cultured cells was prepared by lysis in $\times 1$ SDS-PAGE loading buffer (Ausubel et al, 1992). Approximately $50 \mu \mathrm{g}$ of total cellular protein was electrophoresed in 8\% SDS gels and transferred to Immobilon-P membrane (Millipore, Danvers, Pennsylvania). E2F-4 protein was detected with polyclonal antibody C-20. Proteins were visualized with the enhanced chemiluminescence Western blotting detection system (Amersham Life Science, Arlington Heights, Illinois).

\section{Flow Cytometry Analysis}

Trypsinized cells were washed in ice-cold Tris-saline (10 mm Tris, $\mathrm{pH} 7.0 ; 50 \mathrm{~mm} \mathrm{NaCl}$ ), fixed in $90 \%$ ethanol, and stored for up to 1 week at $4^{\circ} \mathrm{C}$. Cells were washed with phosphate-citric acid buffer (200 $\mathrm{mm} \mathrm{Na}_{2} \mathrm{HPO}_{4} ; 100 \mathrm{~mm}$ citric acid, $\mathrm{pH}$ 7.8) and stained with a solution containing $0.2 \%$ NP40 (Sigma Chemical, St. Louis, Missouri), 7000 units/ml RNase A (Sigma Chemical), and $33 \mathrm{mg} / \mathrm{ml}$ propidium iodide (Sigma Chemical) at $37^{\circ} \mathrm{C}$ for 10 minutes or $4^{\circ} \mathrm{C}$ overnight. Stained nuclei were then analyzed for the percentage of $G_{0} / G_{1}, S, G_{2}-M$, and apoptotic $(<2 N)$ cells using a Becton Dickinson FACScan (San Jose, California) at a laser setting of $15.2 \mathrm{~mW}$ and an 
excitation wavelength of $488 \mathrm{~nm}$. Data were analyzed using Cell Quest version 3.0.1 software (Becton Dickinson). All flow cytometry experiments were repeated five or more times, each in duplicate. Data shown (Fig. 5) indicate representative histograms of a combination of repeated experiments. Where applicable, data from two separately treated conditions were statistically analyzed using Students's $t$ test, and samples were compared based on $p$ values.

\section{Acknowledgement}

We are grateful to Dr. Thomas G. O'Brien (Lankenau Medical Research Center, Wynnewood, Pennsylvania) for providing useful suggestions and editing our manuscript.

\section{References}

Adams PD and Kaelin WJ (1996) The cellular effects of E2F overexpression. Curr Top Microbiol Immunol 208:79-93.

Ausubel F, Brent R, Kingston R, Moore D, Seidman J, Smith $\mathrm{J}$, and Struhl K, editors (1992). Current protocols in molecular biology. New York: John Wiley and Sons, Inc.

Beijersbergen RL, Kerkhoven RM, Zhu L, Carlee L, Voorhoeve PM, and Bernards R (1994). E2F-4, a new member of the E2F gene family, has oncogenic activity and associates with p107 in vivo. Genes Dev 8:2680-2690.

Cartwright $\mathrm{P}$, Muller $\mathrm{H}$, Wagener $\mathrm{C}$, Holm $\mathrm{K}$, and Helin $\mathrm{K}$ (1998). E2F-6: A novel member of the E2F family is an inhibitor of E2F-dependent transcription. Oncogene 17:611623.

Chen C and Okayama $\mathrm{H}$ (1987). High-efficiency transformation of mammalian cells by plasmid DNA. Mol Cell Biol 7:2745-2752.

de la Luna S, Burden MJ, Lee C-W, and La Tangue NB (1996) Nuclear accumulation of the E2F heterodimer regulated by subunit composition and alternative splicing of a nuclear localization signal. J Cell Sci 109:2443-2452.

Ikeda $\mathrm{M}$, Orimo $\mathrm{H}$, Moriyama $\mathrm{H}$, Nakajima E, Matsubara $\mathrm{N}$, Mibu R, Tanaka N, Shimada T, Kimura A, and Shimizu K (1998). Close correlation between mutations of E2F-4 and hMSH3 genes in colorectal cancers with microsatellite instability. Cancer Res 58:594-598.

Herber B, Truss M, Beato M, and Muller R (1994). Inducible regulatory elements in the human cyclin D1 promoter. Oncogene 9:1295-1304.

Krek W, Xu G, and Livingston DM (1995). Cyclin A-kinase regulation of E2F-1 DNA binding function underlies suppression of an S phase checkpoint. Cell 83:1149-1158.

La Thangue NB (1996). E2F and the molecular mechanisms of early cell-cycle control. Biochem Soc Trans 24:54-59.

Li JM, Hu PP, Shen X, Yu Y, and Wang XF (1997). E2F4-RB and E2F4-p107 complexes suppress gene expression by transforming growth factor beta through E2F binding sites. Proc Natl Acad Sci USA 94:4948-4953.
Lindeman GJ, Gaubatz S, Livingston DM, and Ginsberg D (1997). The subcellular localization of E2F-4 is cell-cycle dependent. Proc Natl Acad Sci USA 94:5095-5100.

Liu N, Lucibello FC, Engeland K, and Muller R (1998). A new model of cell cycle-regulated transcription: Repression of the cyclin A promoter by CDF-1 and anti-repression by E2F. Oncogene 16:2957-2963.

Magae J, Wu CL, Illenye S, Harlow E, and Heintz NH (1996). Nuclear localization of DP and E2F transcription factors by heterodimeric partners and retinoblastoma protein family members. J Cell Sci 109:1717-1726.

Muller H, Moroni MC, Vigo E, Petersen BO, Bartek J, and Helin K (1997). Induction of S-phase entry by E2F transcription factors depends on their nuclear localization. Mol Cell Biol 17:5508-5520.

Pearson BE, Nasheuer HP, and Wang TS (1991). Human DNA polymerase alpha gene: Sequences controlling expression in cycling and serum-stimulated cells. Mol Cell Biol 11:20812095.

Sardet C, Vidal M, Cobrinik D, Geng Y, Onufryk C, Chen A, and Weinberg RA (1995). E2F-4 and E2F-5, two members of the E2F family, are expressed in the early phases of the cell cycle. Proc Natl Acad Sci USA 92:2403-2407.

Shan B, Farmer AA, and Lee WH (1996) The molecular basis of E2F-1/DP-1-induced S-phase entry and apoptosis. Cell Growth Differ 7:689-697.

Souza RF, Yin J, Smolinski KN, Zou TT, Wang S, Shi YQ, Rhyu MG, Cottrell J, Abraham JM, Biden K, Simms L, Leggett B, Bova GS, Frank T, Powell SM, Sugimura H, Young J, Harpaz N, Shimizu K, Matsubara N, and Meltzer SJ (1997). Frequent mutation of the E2F-4 cell cycle gene in primary human gastrointestinal tumors. Cancer Res 57:2350-2353.

Vairo G, Livingston DM, and Ginsberg D (1995) Functional interaction between E2F-4 and p130: Evidence for distinct mechanisms underlying growth suppression by different retinoblastoma protein family members. Genes Dev 9:869-881.

Verona R, Moberg K, Estes S, Starz M, Vernon JP, and Lees JA (1997). E2F activity is regulated by cell cycle-dependent changes in subcellular localization. Mol Cell Biol 17:72687282.

Weinberg RA (1996). E2F and cell proliferation: A world turned upside down. Cell 85:457-459.

Weintraub SJ, Prater CA, and Dean DC (1992). Retinoblastoma protein switches the E2F site from positive to negative element. Nature 358:259-261.

Wells JM, Illenye S, Magae J, Wu CL, and Heintz NH (1997). Accumulation of E2F-4. DP-1 DNA binding complexes correlate with induction of dhfr gene expression during the $\mathrm{G} 1$ to S phase transition. J Biol Chem 272:4483-4492.

Yoshitaka T, Matsubara N, Ikeda M, Tanino M, Hanafusa $\mathrm{H}$, Tanaka N, and Shimizu K (1996). Mutations of E2F-4 trinucleotide repeats in colorectal cancer with microsatellite instability. Biochem Biophys Res Commun 227:553-557. 